\title{
Modeling the Variation in the Trajectory of Crosswalk Overflow Violation Pedestrians in China and Countermeasure
}

\author{
Ning-Bo Cao, Zhao-Wei Qu, Xian-Min Song, Li-Ying Zhao, Qiao-Wen Bai, and Rui-Qi Luo \\ College of Transportation, Jilin University, Chang Chun, Jilin 130022, China \\ Correspondence should be addressed to Xian-Min Song; songxm@jlu.edu.cn
}

Received 5 December 2016; Revised 7 April 2017; Accepted 20 July 2017; Published 24 August 2017

Academic Editor: Akemi Gálvez

Copyright (C) 2017 Ning-Bo Cao et al. This is an open access article distributed under the Creative Commons Attribution License, which permits unrestricted use, distribution, and reproduction in any medium, provided the original work is properly cited.

The phenomenon that pedestrians walk outside the crosswalk during green and flashing green is defined as overflow violation, and it is an illegal but common phenomenon in China. Few safety countermeasures can be applied to restrict it. At signalized intersections, one safety issue is the conflicts between turning vehicles and pedestrians. Large variations in overflow pedestrian trajectories lead to widely distributed conflict points. This paper puts forward a model to estimate spatial variations of overflow pedestrian trajectories. By video tracking, only trajectories of overflow violation pedestrians are collected. After analysis, crosswalk geometry, destination, previous passing position, and densities are determined as significant factors of trajectories variations. To simplify modeling process of trajectories variations, this paper assumes that individual trajectory is roughly represented by three feature points on the trajectory. Three feature points are defined as three crossing positions at three cross-sections. Furthermore, Weibull distribution is applied to estimate the spatial distribution of violation pedestrian crossing positions at three cross-sections; then, the spatial distributions of overflow violation pedestrians' trajectories are gained by connecting crossing positions at three cross-sections. Finally, the validation result suggests that the established models are capable of explaining well the spatial maneuver of overflow violation pedestrians' trajectories variations.

\section{Introduction}

Vehicles are restricted to traveling within designated lanes, but pedestrian lateral positions in crosswalk are not confined which results in autonomous pedestrian trajectories. The importance of research on pedestrian behaviors has been well recognized in both the safety field and capacity assessments of road facilities. At signalized intersections in China, overflow violation represents the phenomenon that pedestrians choose to walk outside the crosswalk by taking the shortest path to their destinations. Qu et al. [1] concluded that overflow violation is a common illegal phenomenon in China. As shown in Figure 1, overflow violation has two effects: first, the space for turning vehicles is occupied by overflow violation pedestrians, which increases collisions between pedestrians and turning vehicles; second, pedestrians and turning vehicles compete for priority in greater scope, which leads traffic to be disordered. Therefore, modeling the spatial variation of overflow violation pedestrians' trajectories contributes to improving the design of crosswalk and help guide pedestrians rail position setting; all of these will be conducive to the management of overflow violation pedestrians.

Turner and Penn [2] draw a conclusion that pedestrians tend to take shortest path to their destinations. Bierlaire et al. [3] concluded that pedestrians prefer smooth nonlinear path rather than linear path. Daamen [4] proposed a common framework to model pedestrian motion, and he indicated that pedestrians tend to make decisions according to hierarchical scheme: strategical, tactical, and operational. Antonini et al. [5] made a further study. They presented a dynamics discrete choice framework by using dynamic and individual-based spatial discretization which represents the physical space and indicated that pedestrian short-term behavior served as a response to other pedestrian presence. In the same way, Robin et al. [6] came up with an improved discrete choice model, and then practical data was applied to calibrate the model. They draw a conclusion that pedestrian preferred to keep current direction when heading to the destination. Usher and Strawderman [7] indicated that when collisions with other pedestrians appeared, pedestrians would avoid collisions by 


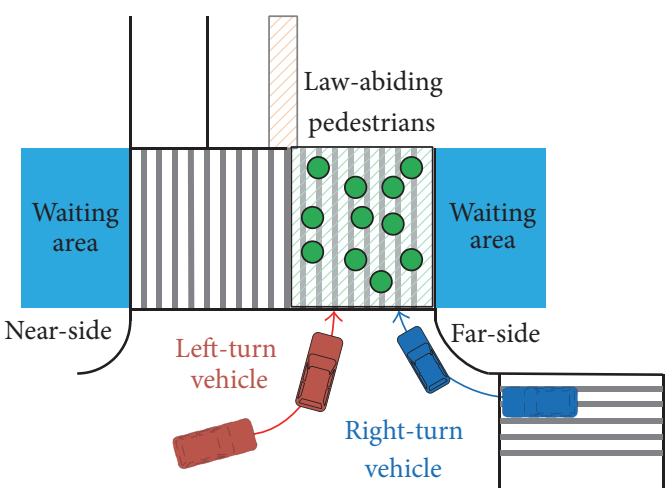

(a) Conflict between turning vehicles and law-abiding pedestrians

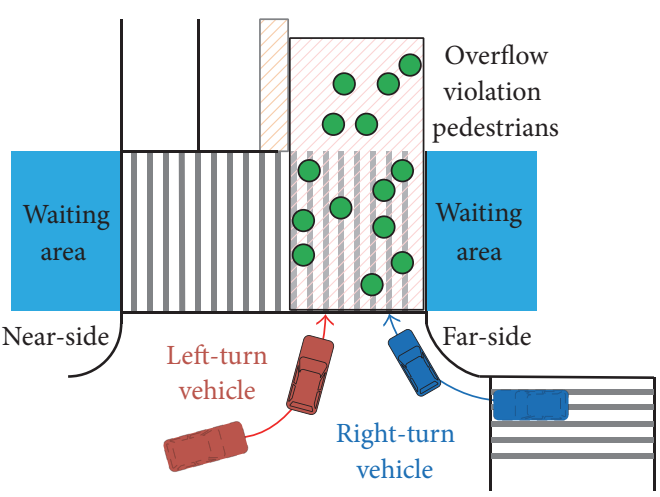

(b) Conflict between turning vehicles and overflow violation pedestrians

FIgURE 1: Conflict between turning vehicles and pedestrians.

changing moving direction or speed. All these studies focused on pedestrians' motion characteristics, but they gave little attention to pedestrian violations, let alone overflow variation of violation pedestrians trajectories.

Nagel et al. [8] reviewed the models of traffic jam formation. They focused on microscopic modeling, that is, coupled differential equations, cellular automata, and so on. Similarly, microscopic simulation and multivariate equations are two common methods applied to model pedestrian behaviors. Microscopic simulation models include social force, cellular automata, and fluid mechanics. Cellular automaton is a cellbased discrete model with discrete time and space units. Zittartz [9] and Blue and Adler [10] established a pedestrian simulation model for pedestrian dynamic evacuation, and they assumed that the boundary of the simulation scenario could not be stepped over. Recently, CA models are used to simulate mixed traffic flow. Pedestrian-vehicle mixed traffic flow is the research highlights. Zhao et al. [11] studied the influence of pedestrians' crossing behavior on the cars and put forward some modified CA rules, such as lane changing, velocity changing, and randomization. Finally, the modified CA model was used to simulate the mixed traffic flow at nonsignalized crosswalk. These studies developed many moving rules to achieve the simulations. As CA models deal with spaces as discretized cells, so it has some shortcomings when simulating unpredictable movements, such as jaywalking. Therefore, continuous space models are put forward. Social force model is one of the common continuous space models. Helbing et al. [12, 13] developed the social force model based on newton's second law of dynamics. The social force model quantifies interactions between pedestrians and outside environment as repulsion and attraction force and then updates pedestrians' positions at each simulation step to simulate pedestrian movements. On the basis of basic SFM, Helbing et al. [14] explored the interactions between pedestrians and cars at crossing sections; besides, they modeled and simulated the interaction behaviors. Pretto et al. [15] combined the rule-based models and force-based models to analyze and model the interactions between pedestrians and cars at crossing points. Recently, the social force model also is used in simulating the mixed traffic flow. Anvari et al. [16] modeled pedestrian-car mixed traffic flow at shared space via rule-based social force model. Zeng et al. [17] proposed a modified social force model to simulate the behaviors of pedestrians at signaled intersection. They indicated that when pedestrians are stepping over the crosswalk boundaries, boundaries would generate an attractive force to draw pedestrians back to crosswalk. Based on these assumptions, they obtained estimated pedestrian trajectories through simulation. These studies only focused on pedestrian overflow caused by the interaction of bidirectional pedestrians, which differs significantly from pedestrian overflow caused by violation. These studies focused on the interactions between pedestrians and cars, and they also rarely consider unpredictable pedestrian violations. Besides, the simulation models are inconvenient to be used in practice directly.

In terms of multivariate equations, different multivariate equations were used to model pedestrian trajectories. A nonparametric pedestrian motion model based on Gaussian process regression was presented by Ellis et al. [18]. They found that pedestrian paths had a stochastic feature which changed with the physical scene structure, the presence of other people, and the time of day. And then, Gaussian process was adopted to reflect the various complexities of different scenes and situations. Papadimitriou et al. [19] summarized the existing pedestrian behaviors studies. They concluded that interactions between pedestrians and vehicles should be studied before modeling pedestrian motions. Brogan and Johnson [20] modeled pedestrian behaviors to describe pedestrian paths planning. They found that every trajectory traced a smooth, circular path. Wei liang et al. [21] built a model to reproduce pedestrian trajectories at signalized crosswalk. They concluded that law-abiding pedestrian trajectories were influenced by intersection geometry, origindestination, previous passing position, and other road users densities. Gorrini et al. [22] pointed out that pedestrians prefer to cut off the crosswalk crossing and tend to choose an oblique path. They concluded that this phenomenon is strictly related to the spatial layout of the side-walks and curbs in the 
TABLE 1: Geometric characteristics of study sites and observation date.

\begin{tabular}{|c|c|c|c|c|c|c|c|}
\hline \multirow{2}{*}{$\begin{array}{l}\text { Objective } \\
\text { crosswalk }\end{array}$} & \multicolumn{2}{|c|}{ Hongqi Street } & \multicolumn{2}{|c|}{ Tongzhi Street } & \multicolumn{2}{|c|}{ Xian Road } & \multirow{2}{*}{$\begin{array}{c}\text { Chongqing } \\
\text { Road } \\
\text { West }\end{array}$} \\
\hline & East & North & East & North & East & South & \\
\hline $\begin{array}{l}\text { Observation time } \\
\text { (p.m) }\end{array}$ & $\begin{array}{c}\text { 1:00-3:00 (2 } \\
\text { days) }\end{array}$ & $\begin{array}{c}\text { 1:00-3:00 (2 } \\
\text { days) }\end{array}$ & $\begin{array}{c}\text { 1:30-3:30 (2 } \\
\text { days) }\end{array}$ & $\begin{array}{c}\text { 1:30-3:30 (2 } \\
\text { days) }\end{array}$ & $\begin{array}{c}\text { 1:00-3:00 (2 } \\
\text { days) }\end{array}$ & $\begin{array}{c}\text { 1:00-3:00 (2 } \\
\text { days) }\end{array}$ & $\begin{array}{c}\text { 1:00-3:00 (2 } \\
\text { days) }\end{array}$ \\
\hline $\begin{array}{l}\text { Crosswalk } \\
\text { width } / \mathrm{m}\end{array}$ & 8 & 6 & 7 & 6 & 10 & 4 & 8 \\
\hline $\begin{array}{l}\text { Crosswalk } \\
\text { length } / \mathrm{m}\end{array}$ & 43 & 23 & 27 & 18 & 44 & 15 & 40 \\
\hline $\begin{array}{l}\text { Distance to } \\
\text { destination } / \mathrm{m}\end{array}$ & 130 & 260 & 90 & 42 & 82 & 30 & 45 \\
\hline $\begin{array}{l}\text { Intersection } \\
\text { angle/deg }\end{array}$ & 90 & 90 & 90 & 90 & 90 & 90 & 90 \\
\hline $\begin{array}{l}\text { Sample size of } \\
\text { overflow } \\
\text { pedestrians }\end{array}$ & 205 & 136 & 172 & 117 & 160 & 160 & 162 \\
\hline $\begin{array}{l}\text { Sample size of all } \\
\text { pedestrians }\end{array}$ & 734 & 591 & 542 & 468 & 669 & 800 & 623 \\
\hline Total & & & & & & & \\
\hline
\end{tabular}

observed scenario. But the mathematical formula of oblique path was not developed.

In summary, although many related studies on pedestrian behaviors were made, not enough attention is paid to pedestrian overflow violation at signalized crosswalk. By using video-processing technology to obtain observed pedestrian trajectories spatiotemporal data, this paper aims to investigate influencing factors of overflow violation pedestrian trajectories variations. For this, a model presenting the variations of overflow violation pedestrian trajectories for different crosswalk geometries is proposed. Spatial distributions of overflow violation pedestrian trajectories are analyzed considering different geometries and distances to the destinations. Variation of overflow violation pedestrian trajectories is modeled to design the position and length of safety barrier, so pedestrian trajectory (not a full path, just samples) is simplified to be represented by three points on pedestrian trajectory. Three points are defined as overflow violation pedestrians' crossing positions at near-side, middleside, and far-side cross-sections.

\section{Study Sites and Data Analysis}

For the sake of analyzing the significance of various influencing factors on the overflow violation pedestrian trajectories, a series of overflow violation pedestrian trajectories data was collected by using video-processing technology at seven signalized crosswalks with different geometric characteristics and traffic environment. All these sites are located in Changchun City, China. The parameters in Table 1 are defined in Figure 2.

2.1. Study Sites. The survey sites have significantly different crosswalk widths, lengths, distances to destinations, and pedestrian volumes. Such a wide range of differences is necessary to rationally study the variations in overflow violation pedestrian trajectories. Besides, all survey sites are located near the commercial centers, and there are bus station, business owners, and shopping malls around them. The software based on video image processing system is applied to extract pedestrian spatiotemporal data. On the basis of pedestrian spatiotemporal data, pedestrian trajectories are acquired. There are sufficient trajectories data to enable a thorough analysis of pedestrian overflow violation. The observation date and crosswalk geometries are shown in Table 1. Date is collected during pedestrian green time.

2.2. Video Collection of Overflow Violation Pedestrian Trajectories. Overflow violation pedestrian trajectories including the crossing positions at different moments are extracted from video data by using method developed by Jiang et al. [23]. Compared with the real trajectory, the trajectory obtained by the method has an error of less than $7 \%$. Pedestrians crossing legally are excluded. The positions of each overflow violation pedestrian are extracted every 0.5 seconds and then their video coordinates are converted to the global coordinates by transformation. Steps of transformation are presented in Figure 2 [1]. (a) Video is shot; (b) pedestrian video coordinates are transformed into global coordinates; (c) pedestrian crossing data is extracted according to the sequence of time and space, and then, pedestrian speed, trajectories, crossing time, and other parameters are obtained; (d) bottom-left corner of the crosswalk is set as the coordinate origin. The point where the right foot is touching the ground is the reference observation point for all pedestrians. All the videos are filmed from high buildings around the intersections so that the observation angle is large enough. Finally, the observed crossing positions are transformed to trajectory. 


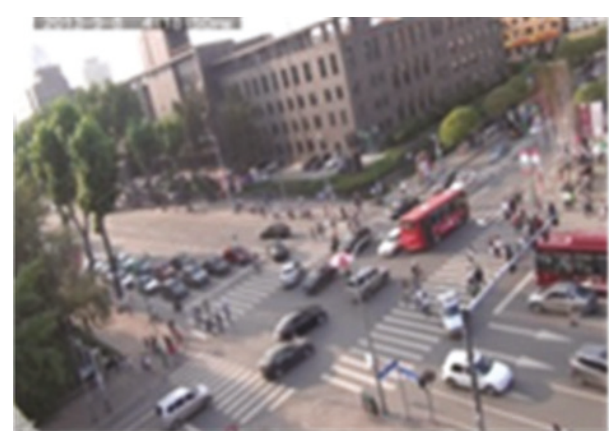

(a)

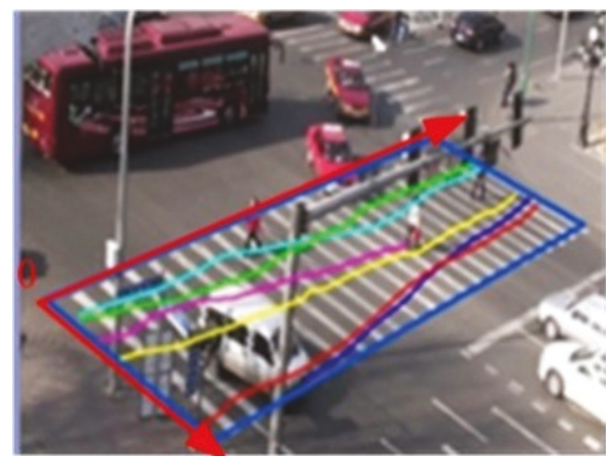

(c)

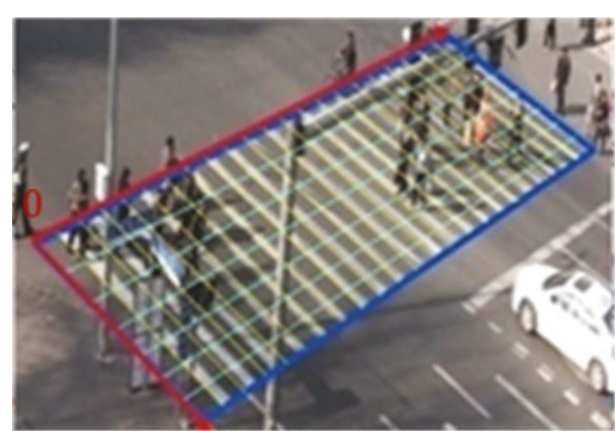

(b)

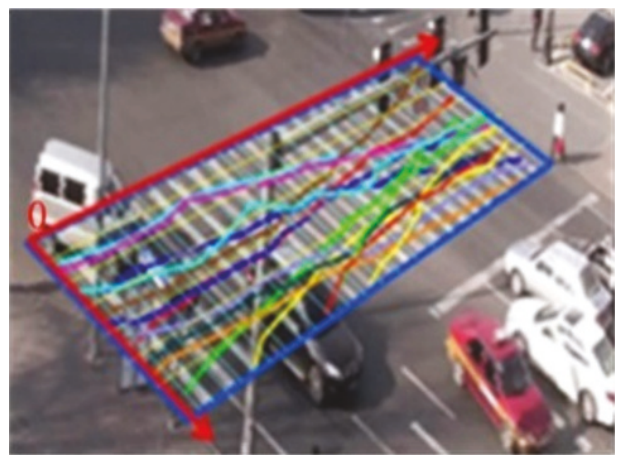

(d)

FIGURE 2: Schematic diagram of coordinates transformation (figure redrawn from Figure 4 of [1]).

2.3. Analysis Method and Definition. Pedestrians modify their trajectories according to traffic environment and interactions with other pedestrians. They tend to choose the shortest path to destinations. In summary, pedestrian trajectories are determined by the perception of traffic environment and occasional elements. According to spatial-temporal database collected by the video, analyzing pedestrian crossing positions at different moments is thought to the best mean to obtain pedestrian trajectories. Because a mountain of work should be done to deal with the data, so it is harder to estimate the inner characteristics of pedestrian trajectories. Studies indicate that pedestrians will not change their current directions unless they meet other pedestrians. Pedestrian crossing positions at near-, middle-, and far-side crosssection determine pedestrian trajectory. The purpose of modeling overflow pedestrian trajectories variation is to serve to the optimization of pedestrian facilities, so pedestrian trajectory determined by three characteristic points is acceptable. As shown in Figure 3(a), near-side means the side where vehicles exist, and far-side is the opposite side. The previous passing position, crosswalk geometry, pedestrian densities, and distance between crosswalk and destination are defined as follows.

As shown in Figure 3(a), crosswalk geometry includes crosswalk width and length. As shown in Figure 3(b), pedestrian crossing positions are defined as the crossing points at three cross-sections. Pedestrian crossing positions at near-, middle-, and far-side cross-section are represented with P1, P2, and P3, respectively. Pedestrian density influences how pedestrians choose their next crossing positions. Observation area is illustrated in Figure 3(c), and pedestrian density is defined as the number of pedestrians divided by the observation area size. Destination represents transport attraction point such as bus station, business owners, or shopping malls. In this paper, the biggest destination is selected as the study focus. The distance between crosswalk and attraction point influences pedestrian crossing behaviors. Pedestrian movement begins from near-side (the paper focuses on overflow violation pedestrian, and overflow violation will not happen at the near-side because vehicles stop at near-side). Overflow violation pedestrian trajectory sketch is shown in Figure 3(b). It is obvious that pedestrians will choose farther crossing position when destination is far from crosswalk, because pedestrians prefer to take the shortest path. As shown in Figure 3(a), the distance between crosswalk and destination is defined as the distance from destination geometric center to crosswalk right boundary.

\section{Data Analysis on Maneuver of Overflow Violation Pedestrian}

3.1. Crosswalk Overflow Violation Pedestrian Trajectories. In previous work, three crosswalks were used to prove the central tendency of overflow pedestrians' trajectories [1]. In order to prove the central tendency, other four crosswalks were added to this paper. When crossing crosswalk, the overflow violation pedestrians' coordinates at every moment were 


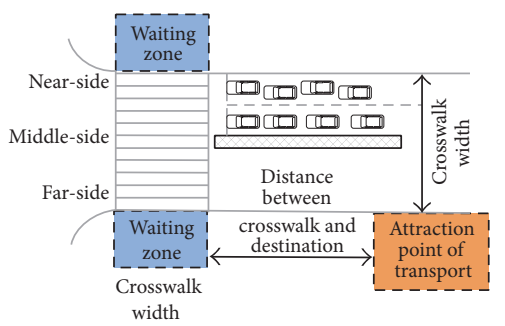

(a) Definition of crosswalk geometry

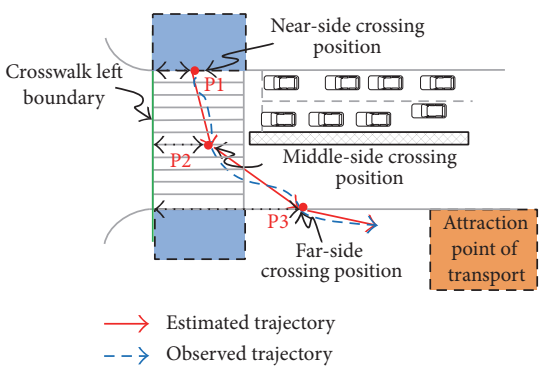

(b) Definition of crossing position

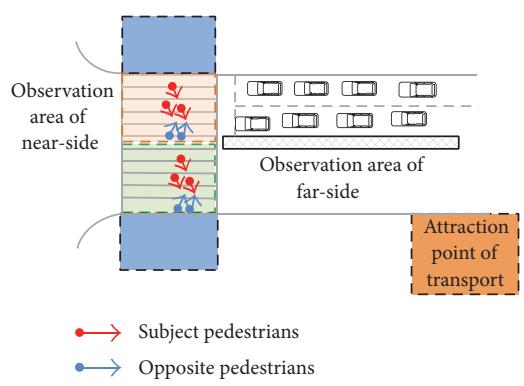

(c) Definition of density detection areas

FIGURE 3: Definition of the influencing factors on pedestrian trajectory.
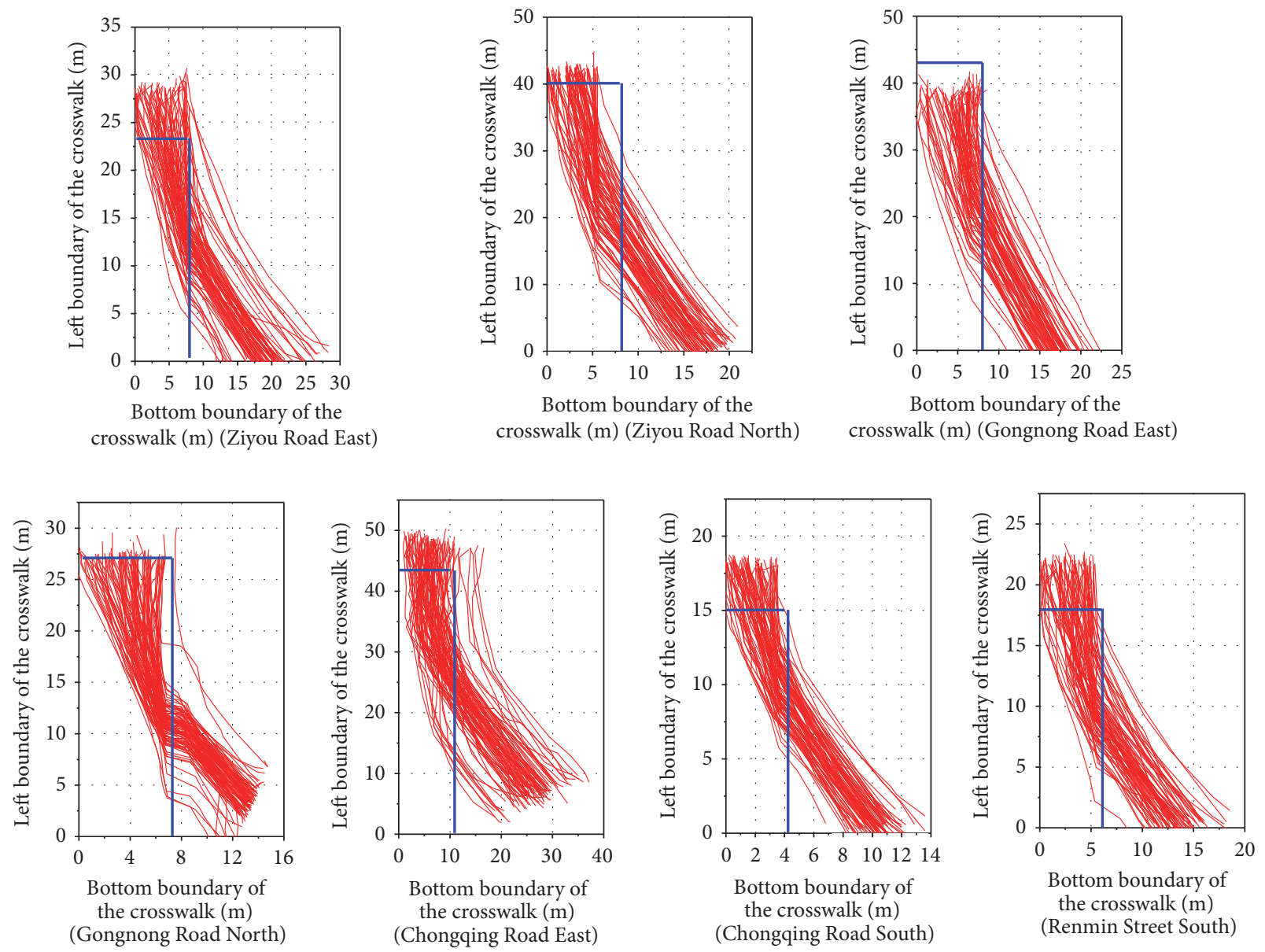

FIgURE 4: Overflow violation pedestrian trajectories variation.

acquired. Based on all positions samples, the trajectories of overflow violation pedestrians were drawn, which are shown in Figure 4. As seen in Figure 4

(1) the overflow pedestrian trajectories variation has central tendency in all investigated crosswalks;

(2) pedestrians begin to go across crosswalk boundary at middle-side cross-section, and, then, they keep on walking outside the crosswalk until finishing crossing at far-side cross-section; it is clear that pedestrian crossing positions at three cross-sections are concentrated in a range; if we want to confine pedestrians to walking on the crosswalk, countermeasure such as pedestrian guard rail set in a certain range at far-side cross-section can achieve the goal; so, a model describing the law of overflow violation pedestrian trajectories variation is quite necessary.

3.2. Distributions of Pedestrian Crossing Positions. The overflow pedestrian trajectory is illustrated in Figure 3(b). It is assumed that pedestrians' trajectories are curved by connecting three points, and three points are defined as the crossing 


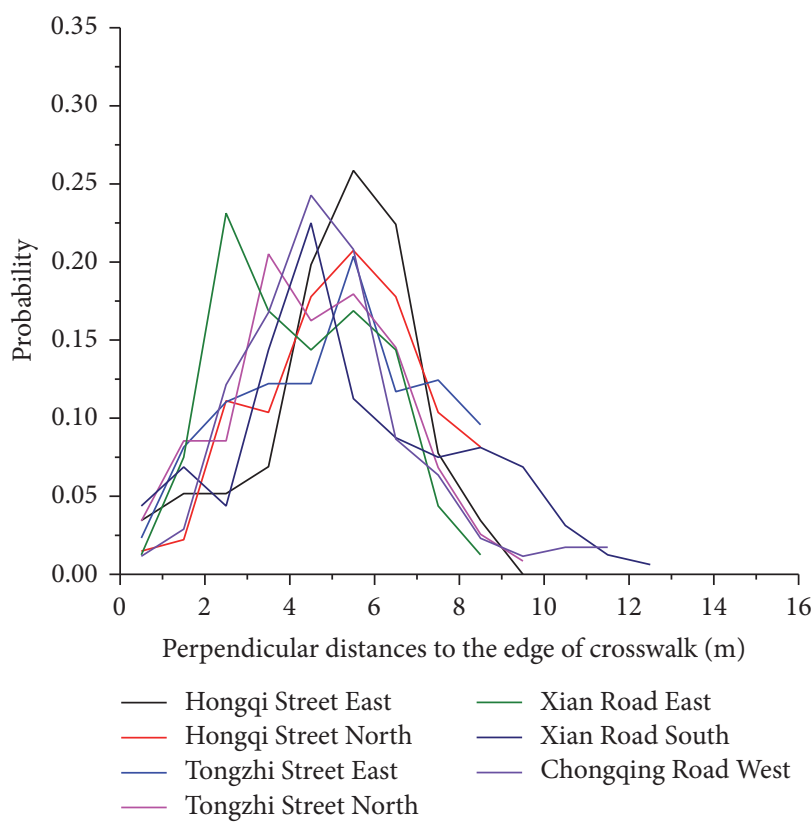

(a) Near-side

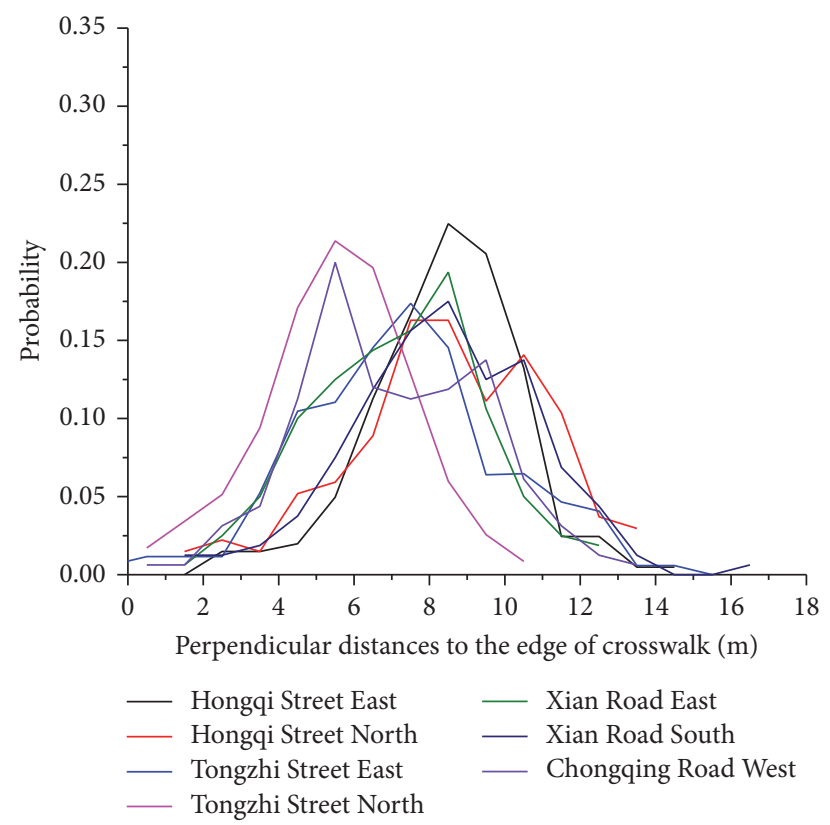

(b) Middle-side

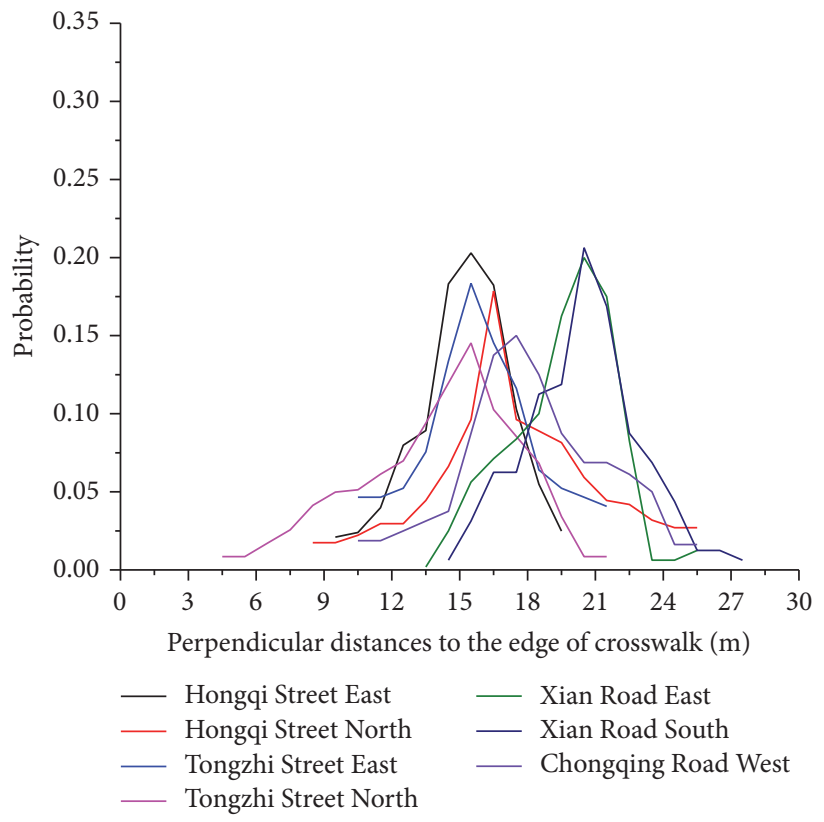

(c) Far-side

FIgURE 5: Pedestrian crossing position distributions at every cross-section.

positions at three cross-sections. So, if we can model the distributions of crossing positions at three cross-sections, the overflow violation pedestrian trajectories variation can be estimated.

Figure 5 shows the raw distributions of pedestrian crossing positions at three cross-sections at seven observed crosswalks. As shown in Figure 5, the peaks of the histograms at near-, middle-, and far-side cross-sections shift to the right side which means that the crossing positions become larger because pedestrians are attracted by destination. For the purpose of illustrating the different characteristics of the shapes of these distributions, appropriate model is needed to be established to flexibly represent the distributions of overflow violation pedestrian crossing positions at three cross-sections.

3.3. Influence Factors of Pedestrian Overflow Violation. Average crossing position is defined as the average value of all overflow violation pedestrians crossing positions at every cross-section in the same green light.

3.3.1. Current Average Crossing Position versus Previous Average Crossing Position. Hongqi Street east crosswalk is taken 


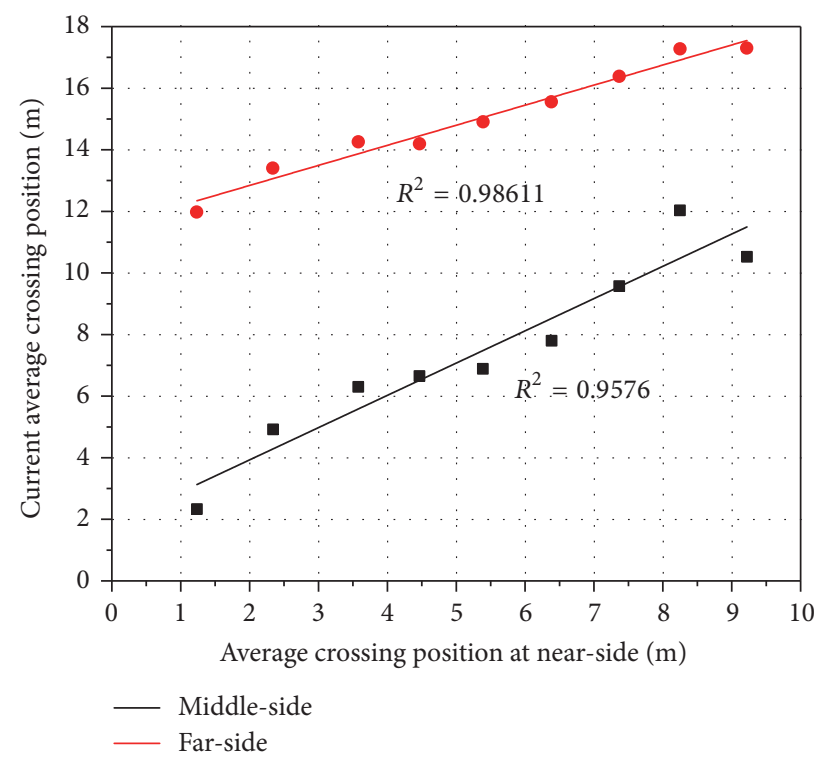

FIGURE 6: Current average crossing position versus previous average crossing position.

as an example to illustrate the influence of average crossing position at previous cross-section on the average crossing position at current cross-section. Linear relationship is adopted to describe the relationship between previous average crossing position and the current average crossing position. Adjusted $R^{2}$ is adopted to test their correlation ship (similar assumption and method are adopted in following analysis of crosswalk geometry and pedestrian density on average passing position). Figure 6 shows the relationships among pedestrian previous average crossing position at nearside cross-section and average crossing positions at middleside and far-side cross-section.

According to adjusted $R^{2}$, with the average crossing position at near-side cross-section becoming larger, the average crossing position at middle-side cross-section significantly increases. Similarly, the average crossing position at farside cross-section also increases with the average crossing position at near-side cross-section becoming larger, which suggests that pedestrian current average crossing position is significantly related to previous average crossing position.

The same method is applied at other study sites. The minimum adjusted $R^{2}$ values at other study sites are 0.66 , which suggests that pedestrian current average crossing position is indeed significantly related to previous average crossing position.

3.3.2. Influence of Crosswalk Width on Average Crossing Position. As shown in Figure 7, the relationship between crosswalk width and average crossing position approximates to linear relationship; there are high $R^{2}$ values at three crosssections, which indicates a significant relationship between crosswalk width and average crossing position. The average crossing position at every cross-section increases with the crosswalk width becoming larger.

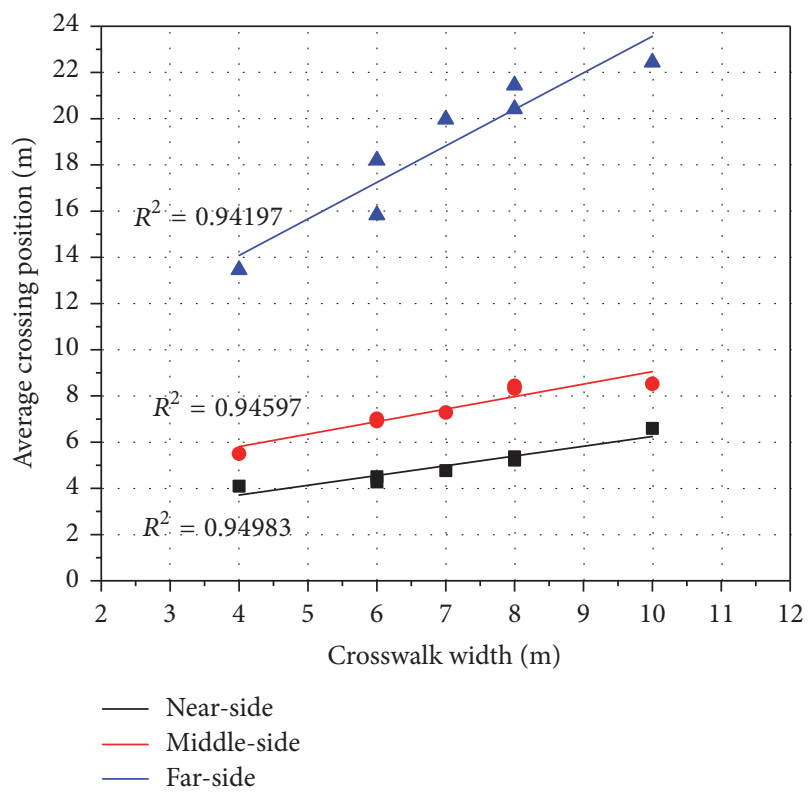

FIgURE 7: Relationship between crosswalk width and average crossing position.

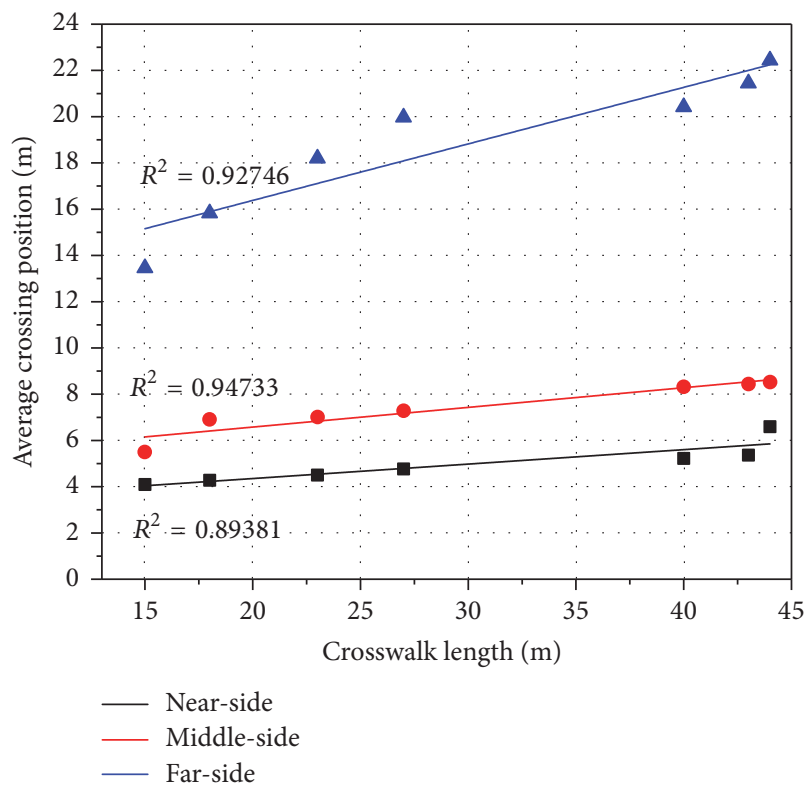

FIGURE 8: Relationship between crosswalk length and average crossing position.

3.3.3. Influence of Crosswalk Length on Average Crossing Position. Similar to the relationship between crosswalk width and average crossing position, Figure 8 shows that there is a significant relationship between crosswalk length and average crossing position. The minimum $R^{2}$ is 0.89 , which indicates that the average crossing position at every crosssection increases with the crosswalk length becoming larger.

3.3.4. Influence of Pedestrian Density on Average Crossing Position. Through regression analysis, $R^{2}$ values of three 


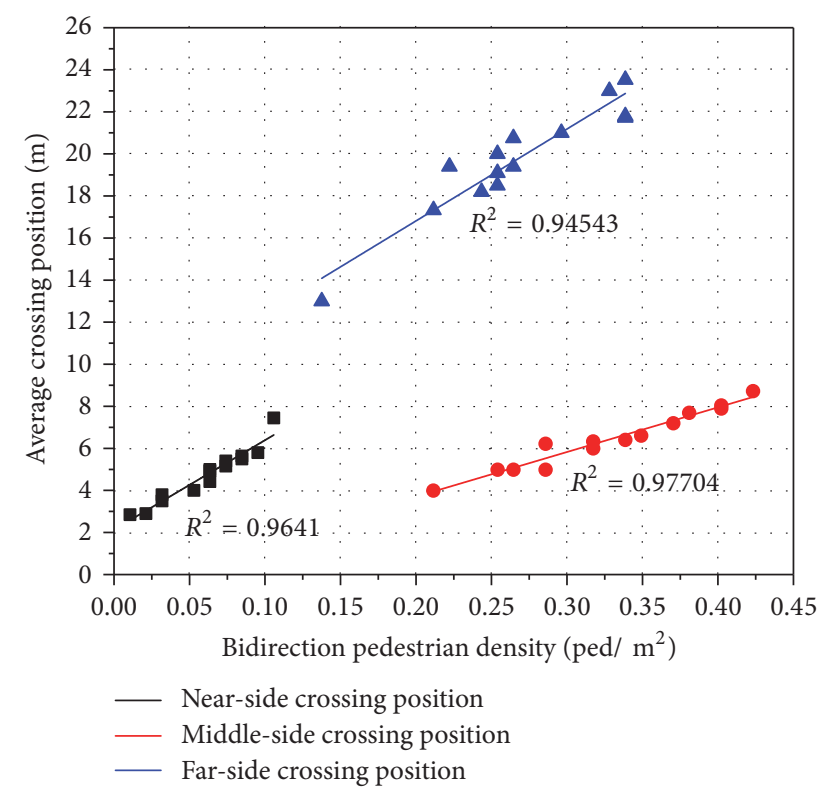

Figure 9: Pedestrian density versus average crossing position.

cross-sections are larger than 0.9. As shown in Figure 9, the relationship between pedestrian density and average crossing position conforms to linear relationship at near-, middle-, and far-side cross-sections, which means that the average crossing position increases with pedestrian density becoming larger.

The same method is applied to analyzing the relationship between pedestrian density and average crossing position at other study sites. The minimum adjusted $R^{2}$ values at other study sites is 0.88 , which suggests that the average crossing position indeed increases with bidirectional pedestrian density becoming larger.

3.3.5. Influence of Distance on Average Crossing Position. It is clear that pedestrians prefer to choose larger crossing position to shorten the distance between his/her current position and destination, which is validated in Figure 10. High $R^{2}$ values suggest a significant relationship between distance and average crossing position. Average crossing positions at three cross-sections increase with distance increasing.

\section{Development and Validation of Trajectory Variation Model}

4.1. Modeling Trajectory Variation. Pedestrian trajectories are curved by connecting three crossing positions at three cross-sections. So, we should model the crossing positions distributions at three cross-sections, and then trajectory variation of crosswalk overflow violation pedestrians is acquired. Walck [24] indicated that Weibull distribution can be used to represent the various consecutive random distribution models by adjusting the shape and scale parameter. So, Weibull distribution is selected to model crossing positions distributions at every cross-section. Based on three established

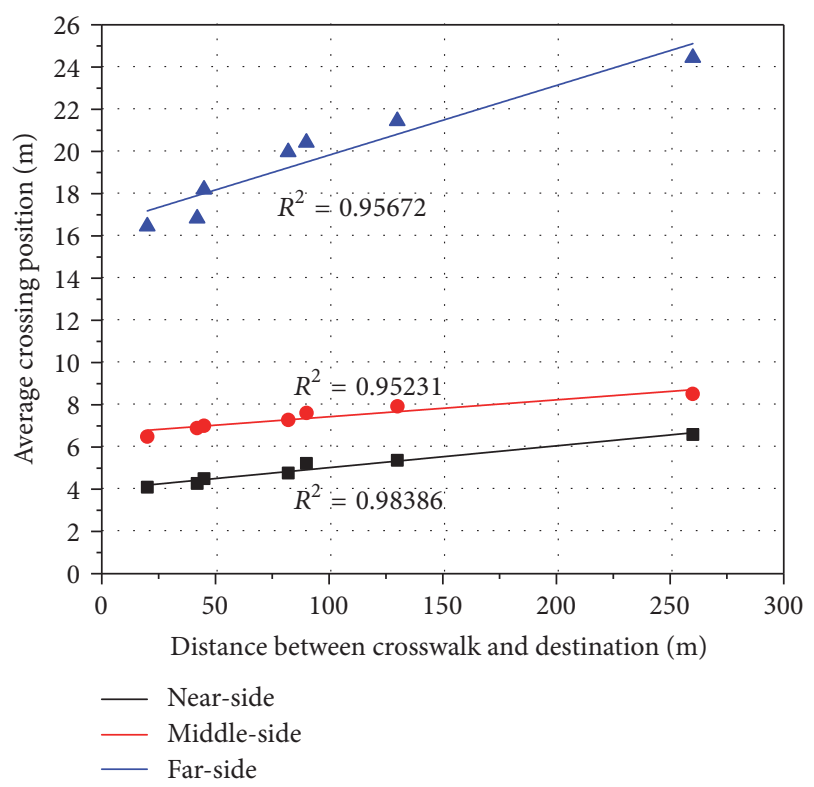

FIGURE 10: Relationship between distance and average crossing position.

crossing positions distribution models, the overflow pedestrian trajectory variation is obtained. The density probability function of Weibull distribution is shown in

$$
f(x, \alpha, \beta)=\frac{\alpha}{\beta}\left(\frac{x}{\beta}\right)^{\alpha-1} e^{-(x / \beta)^{\alpha}},
$$

where $f$ is probability function of Weibull distribution; $\alpha$ is shape parameter which controls the shape of the distribution; $\beta$ is scale parameter which controls the mean value and standard deviation of the distribution.

Before modeling pedestrian crossing positions distributions, two assumptions are made.

(1) Pedestrians keep their directions when walking between each two cross-sections; that is, after changing their directions at three cross-sections, pedestrians will not change their directions until they arrive at next cross-section.

(2) The pedestrian crossing positions distributions at three cross-sections obey Weibull distribution. According to the analyses above, previous average crossing position, crosswalk geometry, destination, and pedestrian density are the key factors influencing the overflow pedestrian trajectories, so they are selected as variables to model pedestrian crossing positions distribution. Shape parameter $\alpha$ and scale parameter $\beta$ of Weibull distribution are modeled as the functions of these five variables, which is shown in (2). The coefficients are estimated by the maximum likelihood method:

$$
\begin{aligned}
\alpha & =f(p, w, l, d, D) \\
& =\lambda_{1,0}+\lambda_{1,1} p+\lambda_{1,2} w+\lambda_{1,3} l+\lambda_{1,4} d+\lambda_{1,5} D, \\
\beta & =f(p, w, l, d, D) \\
& =\lambda_{2,0}+\lambda_{2,1} p+\lambda_{2,2} w+\lambda_{2,3} l+\lambda_{2,4} d+\lambda_{2,5} D,
\end{aligned}
$$


where $p$ is previous average crossing position $(\mathrm{m}) ; w$ is crosswalk width $(\mathrm{m}) ; l$ is crosswalk length $(\mathrm{m}) ; d$ is distance between crosswalk and destination $(\mathrm{m}) ; D$ is pedestrian densities $\left(\mathrm{ped} / \mathrm{m}^{2}\right) ; \lambda_{1,0}, \lambda_{1,1}, \ldots, \lambda_{1, n}$ and $\lambda_{2,0}, \lambda_{2,1}, \ldots, \lambda_{2, n}$ are model coefficients.

At near-side cross-section, the function is shown in (3) and (4). The adjusted $R^{2}$ of $\alpha$ and $\beta$ is 0.65 and 0.68 , respectively.

$$
\begin{aligned}
& \alpha=6.75-0.4 w+0.38 l+0.0022 d-3.55 D \\
& \beta=2.14-0.45 w+0.082 l+0.0026 d+9.45 D
\end{aligned}
$$

At middle-side cross-section, the function is shown in (5) and (6). The adjusted $R^{2}$ of $\alpha$ and $\beta$ is 0.85 and 0.66 , respectively.

$$
\begin{aligned}
\alpha= & 9.03+0.44 p-0.14 w+0.038 l+0.0066 d \\
& -14.16 D \\
\beta= & -3.43+1.28 p+0.61 w-0.075 l-0.0012 d \\
& -4.93 D .
\end{aligned}
$$

At far-side cross-section, the function is shown in (7) and (8). The adjusted $R^{2}$ of $\alpha$ and $\beta$ is 0.95 and 0.63 , respectively.

$$
\begin{aligned}
\alpha= & 19.62+0.59 p+0.75 w-0.072 l-0.0097 d \\
& -37.42 D \\
\beta= & -38.43+4.59 p+4.16 w-0.65 l-0.043 d \\
+ & 28.64 D .
\end{aligned}
$$

The purpose of trajectory variation model is to illustrate pedestrian overflow violation motion and finally put forward countermeasure to confine pedestrian overflow violation.

Four steps are needed to obtain overflow violation pedestrian trajectories variation at crosswalk. Firstly, pedestrian crossing positions distribution at near-side cross-section are calculated according to established equations (3) and (4), and, then, a series of crossing positions obeying the distribution is generated at near-side. Secondly, pedestrian crossing positions distribution at middle-side cross-section are calculated according to established equations (5) and (6). One other thing to note is that previous average passing position is average passing position at near-side. Then, a series of crossing positions obeying the distribution is generated at middle-side. Thirdly, pedestrian crossing positions distribution at far-side cross-section are calculated according to established equations (7) and (8), and previous average passing position is average crossing position at middle-side. Then, a series of crossing positions obeying the distribution model is generated at far-side. Fourthly, pedestrian trajectories are acquired by connecting three crossing positions randomly at three cross-sections; that is, we only get the contour of pedestrian trajectories rather than real and detailed pedestrian trajectories. Finally, overflow violation pedestrian trajectories variation is roughly estimated.
TABLE 2: Variables values for sensitivity analysis.

\begin{tabular}{lcc}
\hline Variable & Value & Cross-section \\
\hline Previous average crossing position & $6.72[\mathrm{~m}]$ & Middle-side \\
Pedestrian density & $0.33\left[\mathrm{ped} / \mathrm{m}^{2}\right]$ & \\
\hline
\end{tabular}

Model validation is conducted to confirm whether established model can well represent overflow pedestrian trajectories variation at signalized crosswalk. By comparing observed pedestrian trajectories variation and estimated results, validation is conducted at Hongqi Street east crosswalk based on pedestrian crossing positions distributions at three crosssections.

Hongqi Street east crosswalk is selected as the validation site, because it has longer crosswalk length, width, pedestrian volumes, and attraction point. The crosswalk geometry is shown in Table 1. Those pedestrians who finish crossing legally in crosswalk entirely are not taken into account when recording data. Because the paper focus is on overflow violation pedestrians who finish crossing outside the crosswalk by the shortest path illegally.

4.2. Estimated Trajectories Variation versus Observed Trajectories Variation. As shown in Figure 11, observed pedestrian trajectories variation and estimated pedestrian trajectories variation are compared. After using two sample $t$-tests for the difference of crossing positions at three cross-sections at a $95 \%$ confidence level, there is no significant difference according to analysis result.

By comparing the probability and cumulative probability of observed and estimated crossing positions at three crosssections, it is obvious that the distributions of estimated crossing positions agree with observed ones better at the nearand middle-side cross-sections than that at far-side crosssection. It is due to the fact that pedestrians have more choices at far-side cross-section when pedestrians walk outside the crosswalk; in addition, turning vehicles have more influences on pedestrian motion, and pedestrian movements are more stochastic. It means that estimated pedestrian trajectories variation agrees with the observed pedestrian trajectories variation well.

\section{Sensitivity Analysis of Parameters}

Hongqi Street east crosswalk is selected as the validation site. Sensitivity analysis enables us to understand variation of established models with the change of five variables. Sensitivities of previous average crossing position, crosswalk width and length, pedestrian density, and distance are analyzed. Other variables values are shown in Table 2 when the objective variable varies in a certain range.

5.1. Sensitivity of Previous Average Crossing Position. Sensitivity analysis of previous average crossing position is conducted under conditions that average crossing positions at near-side are set to be a set of values, such as $4 \mathrm{~m}, 5 \mathrm{~m}, 6 \mathrm{~m}, 7 \mathrm{~m}$, $8 \mathrm{~m}, 9 \mathrm{~m}$, and $10 \mathrm{~m}$. The computation results are shown in 

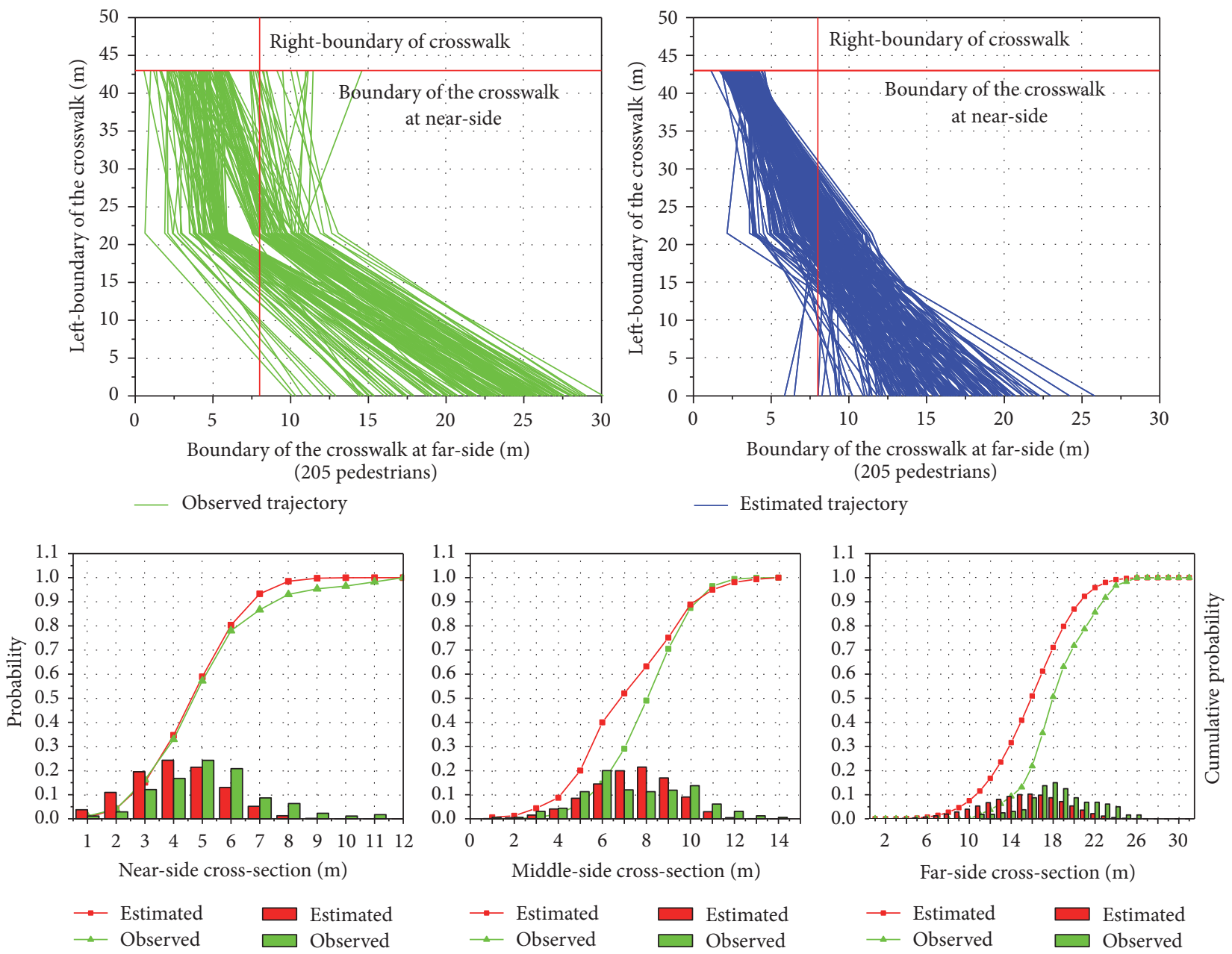

FIGURE 11: Comparison of observed and estimated pedestrian trajectories variation.

Figure 12(a). It is clear that crossing positions at middle-side cross-section tend to be more and more concentrated with the average crossing position at near-side cross-section becoming larger, which suggests that pedestrians prefer to determine their crossing positions according to the average crossing position at previous crossing position. If pedestrian crossing position at near-side cross-section is far always from the left edge of crosswalk, pedestrians have fewer choices of crossing positions at middle-side cross-section. Similarly, if pedestrian crossing position at near-side cross-section is close to the left edge of crosswalk, they have more choices of crossing positions at middle-side cross-section.

5.2. Sensitivity of Crosswalk Width. In a similar way, crosswalk widths are set as $4 \mathrm{~m}, 5 \mathrm{~m}, 6 \mathrm{~m}, 7 \mathrm{~m}$, and $8 \mathrm{~m}$, and other variables are set as shown in Tables 1 and 2 . The crossing positions at middle cross-section are used to conduct sensitivity analysis of crosswalk width. Conclusion is drawn from Figure 13(a) that pedestrians tend to choose larger crossing position at middle cross-section when facing wider crosswalk. In addition, crossing positions at middle-side cross-section tend to be more and more diverging with the crosswalk becoming wider, because pedestrians have more choices of crossing positions at middle cross-section at a wider crosswalk.

5.3. Sensitivity of Crosswalk Length. Similar to the sensitivity of crosswalk width, crosswalk lengths are set as $20 \mathrm{~m}, 25 \mathrm{~m}$, $30 \mathrm{~m}, 35 \mathrm{~m}, 40 \mathrm{~m}, 45 \mathrm{~m}$, and $50 \mathrm{~m}$, as shown in Figure 14(a). It is obvious that pedestrians tend to walk farther from the edge of the crosswalk as crosswalk length becomes larger, because pedestrians prefer to choose the shortest path.

5.4. Sensitivity of Pedestrian Density. Because subject pedestrians meet opposite pedestrians at middle-side crosssection, so pedestrian density is the density of bidirectional pedestrian which is set to be $0 \mathrm{ped} / \mathrm{m}^{2}, 0.15 \mathrm{ped} / \mathrm{m}^{2}$, $0.30 \mathrm{ped} / \mathrm{m}^{2}, 0.45 \mathrm{ped} / \mathrm{m}^{2}$, and $0.6 \mathrm{ped} / \mathrm{m}^{2}$ for analyzing. From Figure 15(a), we know that pedestrians will choose the crossing positions farther from the left edge of crosswalk with pedestrian density becoming larger. This is due to pedestrians having to choose farther positions to avoid collisions with other pedestrians.

5.5. Sensitivity of Distance between Crosswalk and Destination. Distances between crosswalks and destinations are set as 


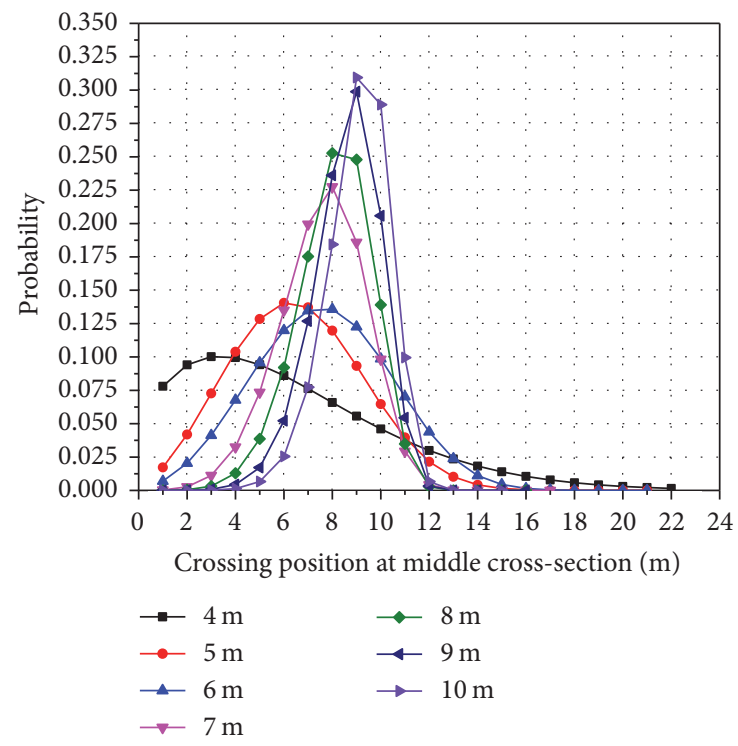

(a)

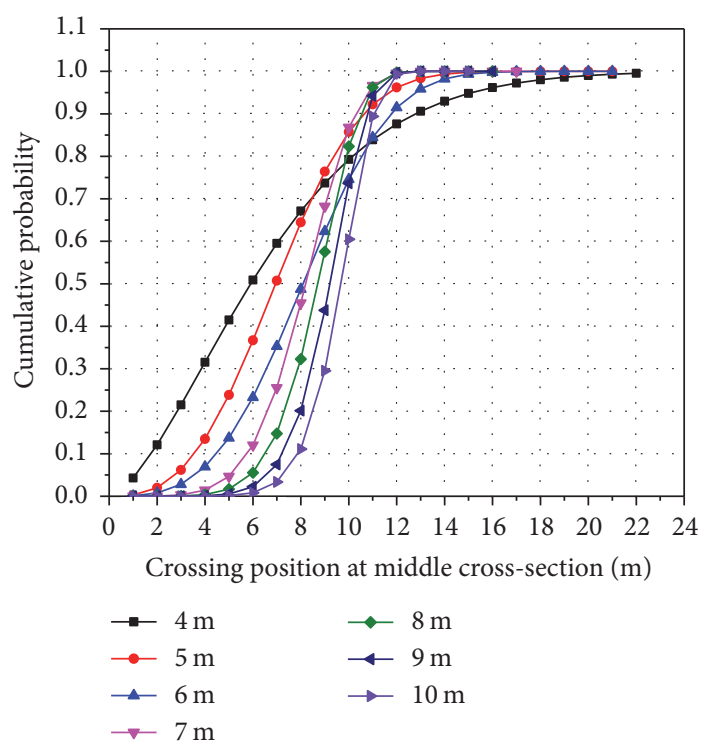

(b)

FIGURE 12: The sensitivity of previous average passing position.

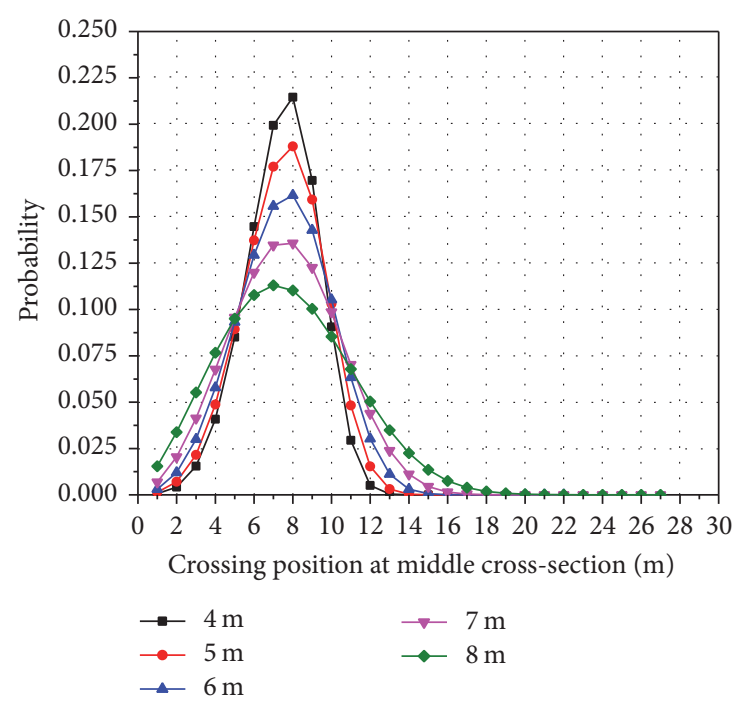

(a)

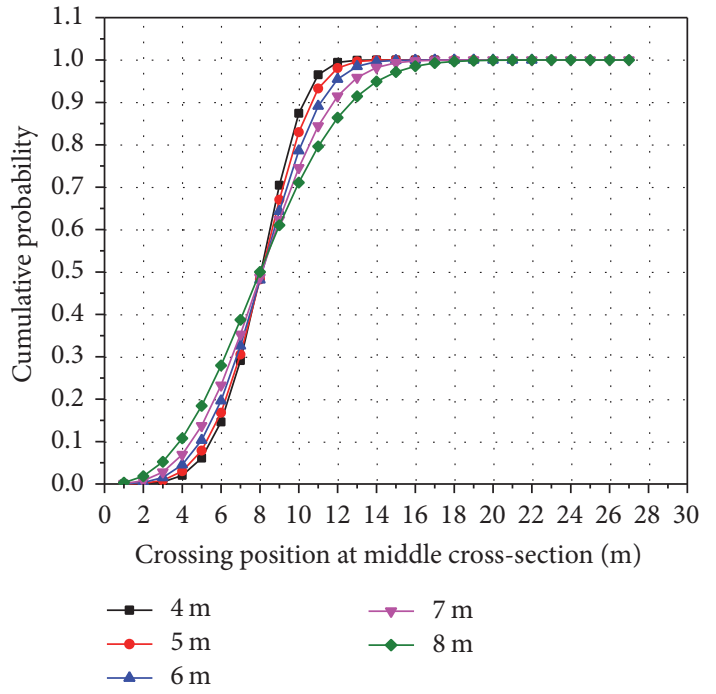

(b)

Figure 13: The sensitivity of crosswalk width.

$50 \mathrm{~m}, 100 \mathrm{~m}, 150 \mathrm{~m}$, and $200 \mathrm{~m}$, and the middle cross-section is selected for analyzing. As shown in Figure 16(a), when pedestrians face farther destination, they tend to choose the shortest path to head to the destination. That is, pedestrian chooses larger crossing position to shorten his/her path.

\section{Countermeasure for Overflow Violation Based on Trajectories Variation}

6.1. Countermeasure. At those intersections where traffic attractive points exist, pedestrians overflow violation is a common behavior. In previous work, countermeasures were put forward to restrict overflow violations [1]. Based on the overflow violation pedestrian trajectories variation, improved method is put forward. New method aims at restricting pedestrians to walking on crosswalk, which guarantees the safety of pedestrians when crossing the crosswalk.

Overflow violation pedestrian trajectories variation has central tendency. Considering this characteristic, countermeasure is suggested to alleviate the overflow violation, as shown in Figure 17. The safety barrier is effective in preventing pedestrians from walking outside crosswalk, and pedestrians have to finish crossing legally rather than crossing by the shortest path illegally.

6.2. Effectiveness of Countermeasure. Taking Hongqi Street east crosswalk as an example, we set safety barrier with different length, and the frequencies of overflow violation 


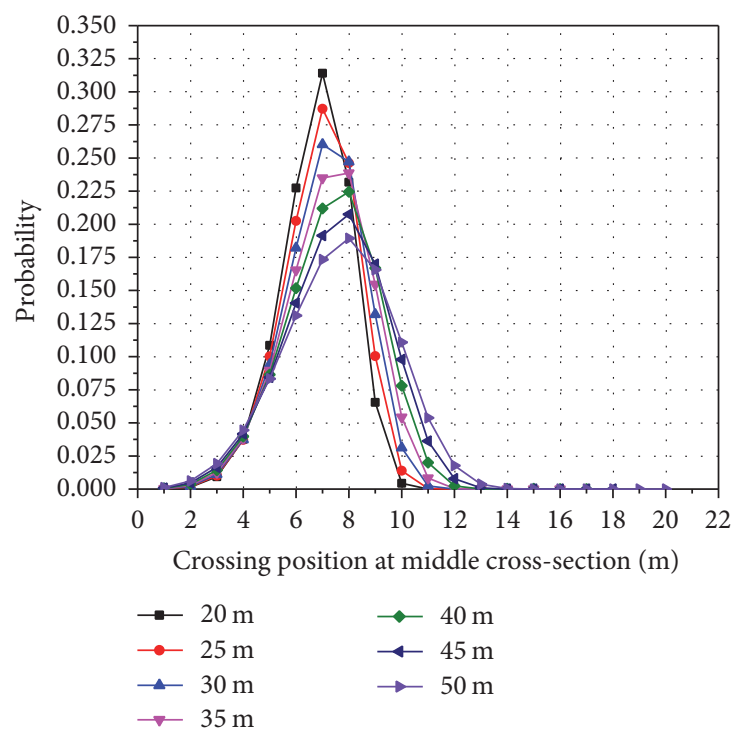

(a)

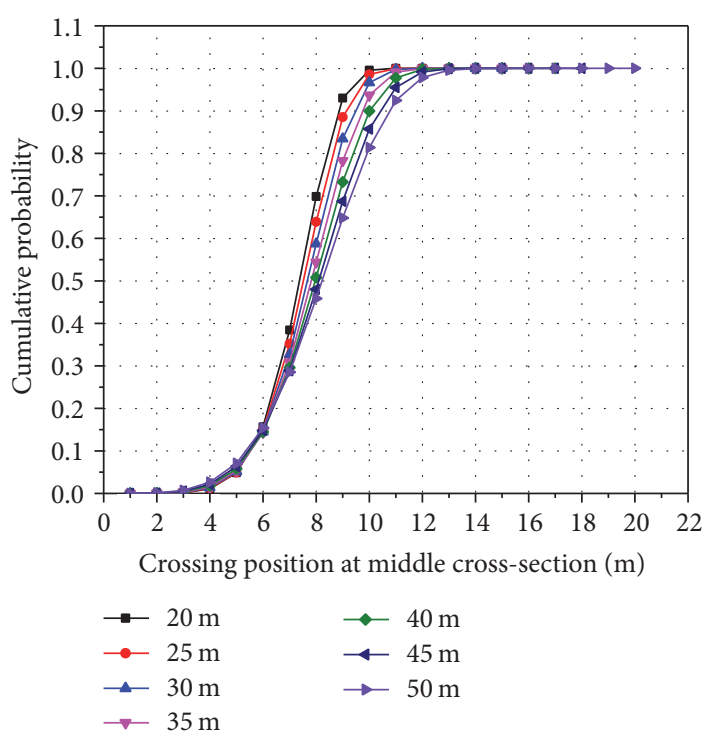

(b)

Figure 14: The sensitivity of crosswalk length.

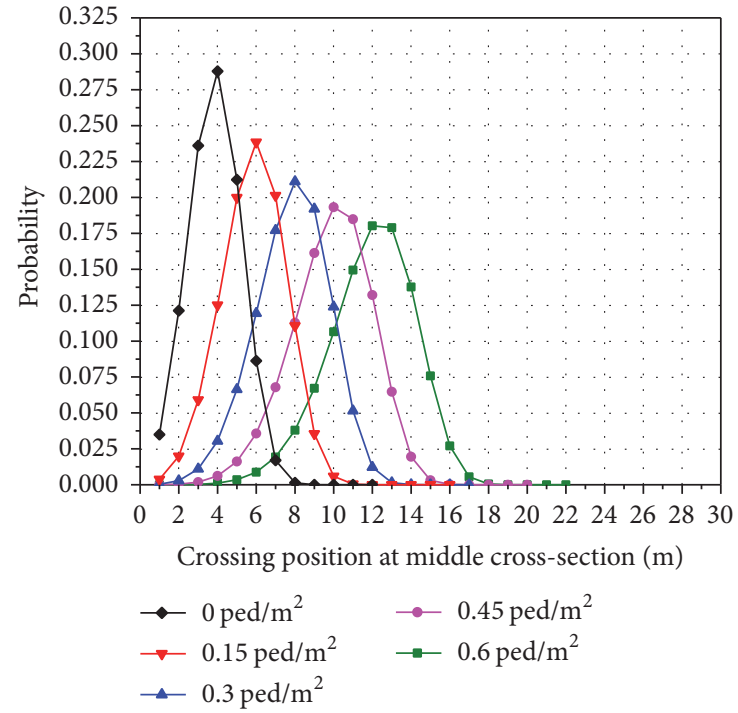

(a)

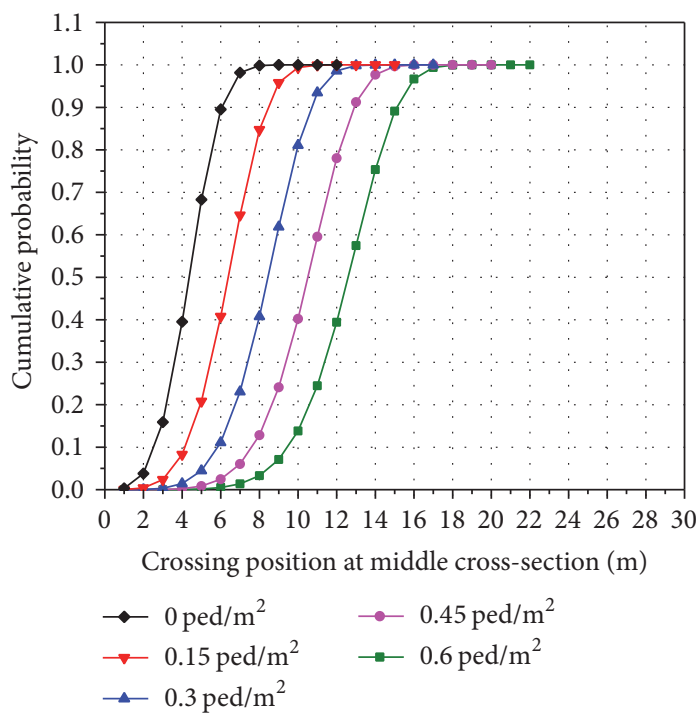

(b)

Figure 15: The sensitivity of pedestrian density.

were recorded. Besides, we estimated the frequencies of overflow violation based on the overflow violation pedestrian trajectories variation model when safety barrier length takes different value. Safety barrier is adopted to prevent the overflow violation from occurring, and the observed frequency of overflow violation and estimated frequency of overflow violation are compared, and the comparison results are shown in Figure 18. As shown in Figure 18, the frequency of overflow violation reduces to $85 \%$, which means that $15 \%$ overflow pedestrians are limited to walking on crosswalk. The safety barrier length is 8 meters according to the observed data and the safety barrier length is 9 meters according to the estimated data based on the proposed model. With safety barrier length increasing, more and more pedestrians are limited to walking on crosswalk. According to the analysis above, when the safety barrier is set, the crossing positions of those overflow pedestrians will be located on the crosswalk.

When the safety barrier length is 12.5 meters, the observed and estimated frequencies of overflow reduce to $50 \%$, and half of overflow pedestrians no longer walk outside the crosswalk. And then, the observed and estimated frequencies of overflow reduce to $5 \%$ when the safety barrier lengths are 17 meters and 15 meters, respectively. When the safety barrier length goes on increasing to 19 meters and 17 meters, the overflow almost no 


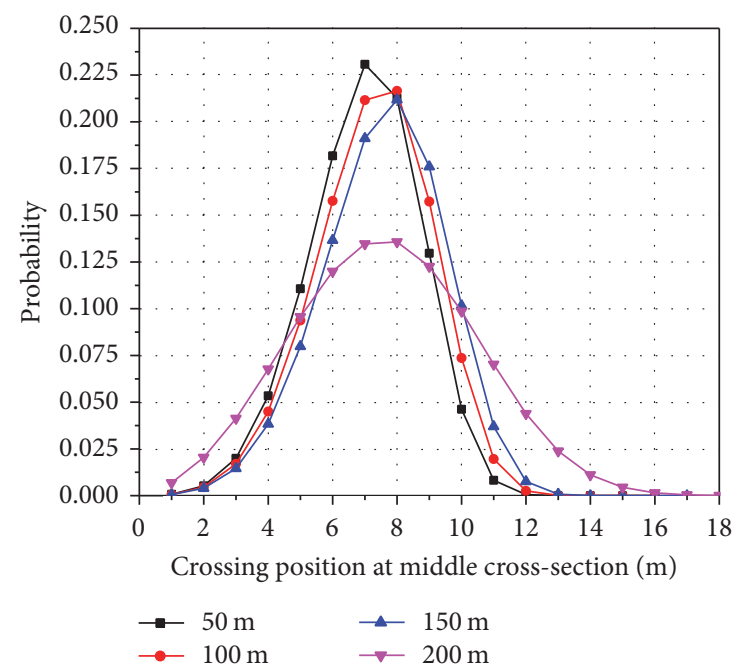

(a)

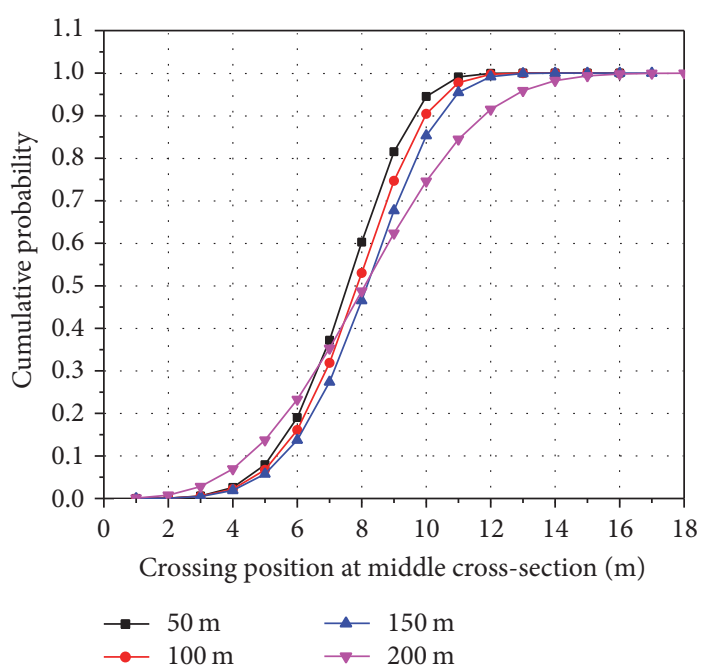

(b)

FIGURE 16: The sensitivity of distance between crosswalk and destination.

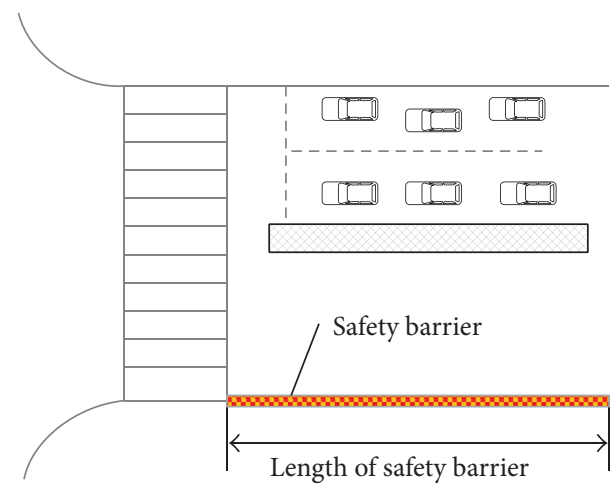

FIGURE 17: Countermeasure for overflow violation (figure redrawn from Figure 8 of [1]).

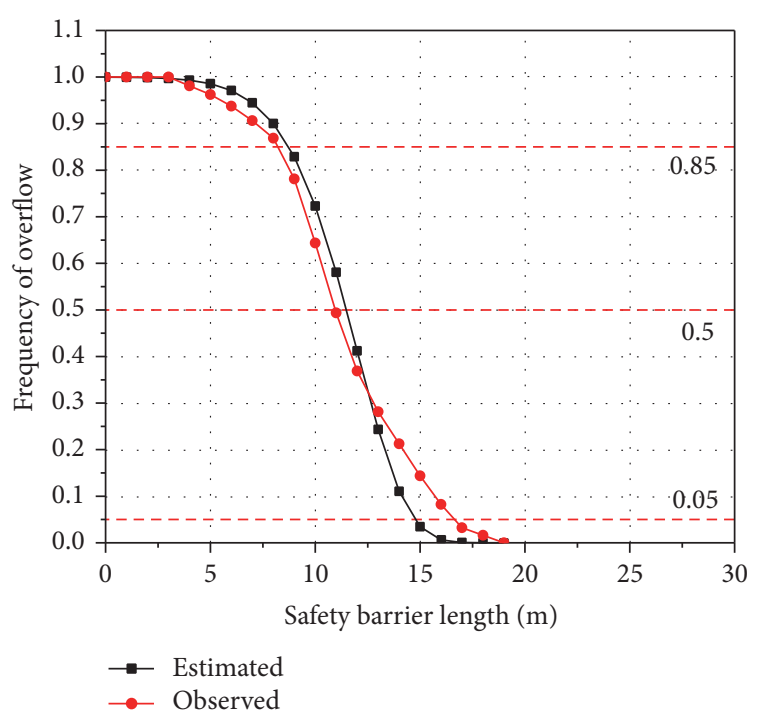

FIGURE 18: Frequency of the overflow violation after setting countermeasure. longer occurs according to both observed data and estimated data.

It is clear that most of overflow pedestrians could be confined to walking on the crosswalk when the safety barrier length reaches a certain value, which suggests that the countermeasure is effective. The largest length of safety barrier can be estimated according to proposed overflow violation pedestrian trajectories variation model. With the safety barrier length increasing, more and more overflow pedestrians are limited to walking on the crosswalk.

\section{Conclusions}

In the previous work, the overflow characteristics were analyzed, and the central tendency of overflow pedestrians' trajectories was proved [1]. On the basis of the previous work, the paper focuses on trajectories variation of overflow violation pedestrians. Overflow violation pedestrian trajectories variation model is developed to illustrate overflow violation maneuver, and finally it put forward a countermeasure to confine overflow violation pedestrian to walking on the crosswalk. The overflow violation pedestrian trajectories variation is analyzed and modeled as a function containing three crossing positions distribution models at three cross-sections considering previous average crossing position, geometry, distance, and pedestrian density.

After analyzing the influences of previous average crossing position, crosswalk geometry, distance, and pedestrian density on pedestrian current crossing position, Weibull distribution is selected to model the distributions of pedestrian crossing positions at three cross-sections. Based on the distributions of pedestrian crossing positions, overflow violation pedestrian trajectories variation is obtained, but one other thing to note is that we only get the contour of pedestrian trajectories rather than real and detailed pedestrian trajectories. The established overflow violation pedestrian trajectories variation model is unfit for describing the detailed 
pedestrian motion and the interactions with other road users, but the model can be used as to design pedestrian safety facility. Based on proposed model, countermeasures for overflow violation are suggested to alleviate overflow violation. It is found that most of overflow pedestrians could be confined to walking on the crosswalk when the safety barrier length reaches certain value, which suggests that the countermeasure is effective. The largest length of safety barrier can be estimated according to overflow violation pedestrian trajectories variation calculated by the proposed model. Furthermore, the proposed model gives guidance on how to prohibit pedestrians overflow violation in China.

However, several limitations of the established model should not be ignored. The influences of turning vehicles and phase are not considered. For example, pedestrians tend to choose a larger crossing position at the end of the green light at near-side cross-section. That is our future research.

\section{Conflicts of Interest}

The authors declare that they have no conflicts of interest.

\section{Acknowledgments}

This research is funded by the National Natural Science Foundation of China (nos. 51278520 and 51278220).

\section{References}

[1] Z.-W. Qu, N.-B. Cao, Y.-H. Chen et al., "Overflow characteristics of pedestrian crossing at signalized intersections," Jilin Daxue Xuebao (Gongxueban)/Journal of Jilin University (Engineering and Technology Edition), vol. 46, no. 5, pp. 1432-1438, 2016.

[2] A. Turner and A. Penn, "Encoding natural movement as an agent-based system: An investigation into human pedestrian behaviour in the built environment," Environment and Planning B: Planning and Design, vol. 29, no. 4, pp. 473-490, 2002.

[3] M. Bierlaire, G. Antonini, and M. Weber, "Behavioral dynamics for pedestrians," in Proceedings of the 10th International Conference on Travel Behavior Research, Lucerne, Switzerland, 2003.

[4] W. Daamen, Modelling passenger flows in public transport facilities. Doctoral dissertation [Doctoral, thesis], Delft University of Technology, Delft, Netherlands, 2004.

[5] G. Antonini, M. Bierlaire, and M. Weber, "Discrete choice models of pedestrian walking behavior," Transportation Research Part B: Methodological, vol. 40, no. 8, pp. 667-687, 2006.

[6] T. Robin, G. Antonini, M. Bierlaire, and J. Cruz, "Specification, estimation and validation of a pedestrian walking behavior model," Transportation Research Part B: Methodological, vol. 43, no. 1, pp. 36-56, 2009.

[7] J. M. Usher and L. Strawderman, "Simulating operational behaviors of pedestrian navigation," Computers \& Industrial Engineering, vol. 59, no. 4, pp. 736-747, 2010.

[8] K. Nagel, P. Wagner, and R. Woesler, "Still flowing: Approaches to traffic flow and traffic jam modeling," Operations Research, vol. 51, no. 5, pp. 681-837, 2003.

[9] C. B. K. K. A. S. J. Zittartz, "Simulation of pedestrian dynamics using a two-dimensional cellular automaton," Physica A, vol. 295, pp. 507-525, 2001.
[10] V. J. Blue and J. L. Adler, "Cellular automata microsimulation for modeling bi-directional pedestrian walkways," Transportation Research Part B: Methodological, vol. 35, no. 3, pp. 293-312, 2001.

[11] H.-T. Zhao, S. Yang, and X.-X. Chen, "Cellular automata model for urban road traffic flow considering pedestrian crossing street," Physica A: Statistical Mechanics and its Applications, vol. 462, pp. 1301-1313, 2016.

[12] D. Helbing and P. Molnár, "Social force model for pedestrian dynamics," Physical Review E, vol. 51, no. 5, pp. 4282-4286, 1995.

[13] D. Helbing, I. Farkas, and T. Vicsek, "Simulating dynamical features of escape panic," Nature, vol. 407, no. 6803, pp. 487490, 2000.

[14] D. Helbing, R. Jiang, and M. Treiber, "Analytical investigation of oscillations in intersecting flows of pedestrian and vehicle traffic," Physical Review E: Statistical, Nonlinear, and Soft Matter Physics, vol. 72, no. 4, Article ID 046130, 2005.

[15] Pretto C. O., Cybis H. B. B., and A. Jacobsen, "A multi-layer simulation model for vehicle and pedestrian interaction[C]," in Proceedings of the Transportation Research Board 90th Annual Meeting, pp. 11-3833, 2011.

[16] B. Anvari, M. G. H. Bell, A. Sivakumar, and W. Y. Ochieng, "Modelling shared space users via rule-based social force model," Transportation Research Part C: Emerging Technologies, vol. 51, pp. 83-103, 2015.

[17] W. Zeng, P. Chen, H. Nakamura, and M. Iryo-Asano, "Application of social force model to pedestrian behavior analysis at signalized crosswalk," Transportation Research Part C: Emerging Technologies, vol. 40, pp. 143-159, 2014.

[18] D. Ellis, E. Sommerlade, and I. Reid, "Modelling pedestrian trajectory patterns with Gaussian processes," in Proceedings of the 2009 IEEE 12th International Conference on Computer Vision Workshops, ICCV Workshops 2009, pp. 1229-1234, Kyoto, Japan, October 2009.

[19] E. Papadimitriou, G. Yannis, and J. Golias, "A critical assessment of pedestrian behaviour models," Transportation Research Part F: Traffic Psychology and Behaviour, vol. 12, no. 3, pp. 242-255, 2009.

[20] D. C. Brogan and N. L. Johnson, "Realistic human walking paths," in Proceedings of the 16th International Conference on Computer Animation and Social Agents, pp. 94-101, New Brunswick, NJ, USA.

[21] Z. Wei liang, C. Peng, N. Hideki, and A. Miho, "Modeling Pedestrian Trajectory for Safety Assessment at Signalized Crosswalks," in Proceedings of the 10th International Conference of the Eastern Asia Society for Transportation Studies, Taipei, Taiwan, 2013.

[22] A. Gorrini, G. Vizzari, and S. Bandini, “Towards Modelling Pedestrian-Vehicle Interactions: Empirical Study on Urban Unsignalized Intersection," https://arxiv.org/abs/1610.07892.

[23] S. Jiang, D. H. Wang, and Qu Z. W., "Extraction and Calibration of Trajectory Characteristics of Vehicles at Intersections[J]," Journal of Southwest Jiao tong University, pp. 5-9, 2012.

[24] C. Walck, "Hand-book on statistical distributions for experimentalists," Tech. Rep. Internal Report SUF-PFY/96-01, University of Stockholm, 2007. 


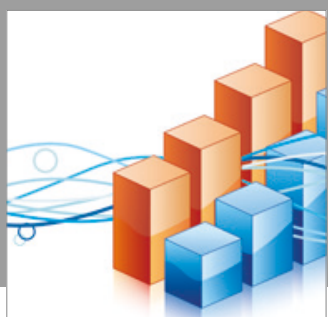

Advances in

Operations Research

vatersals

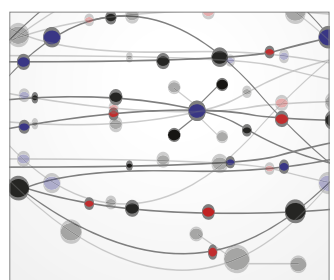

\section{The Scientific} World Journal
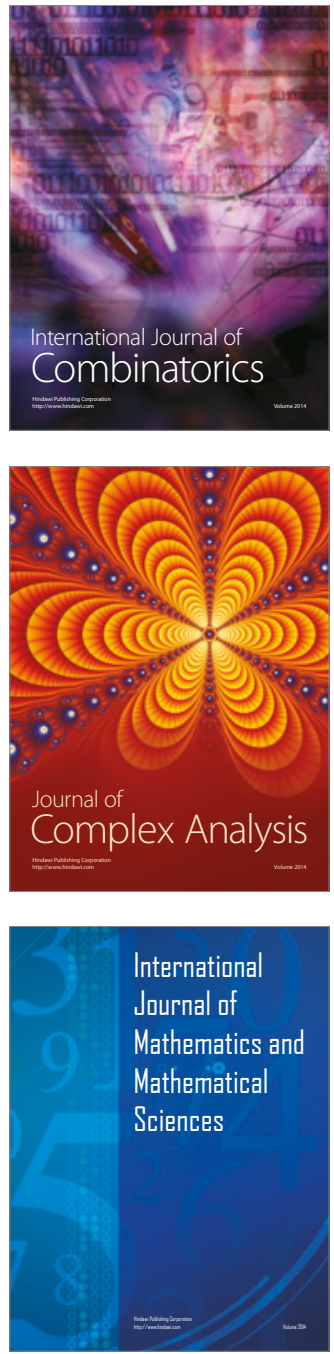
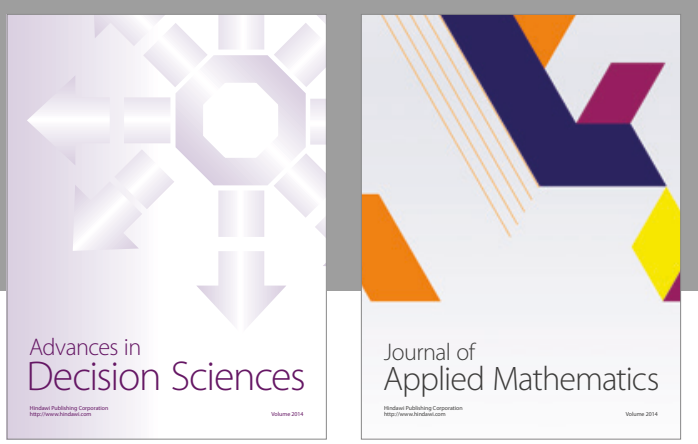

Algebra

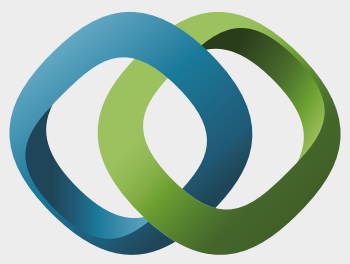

\section{Hindawi}

Submit your manuscripts at

https://www.hindawi.com
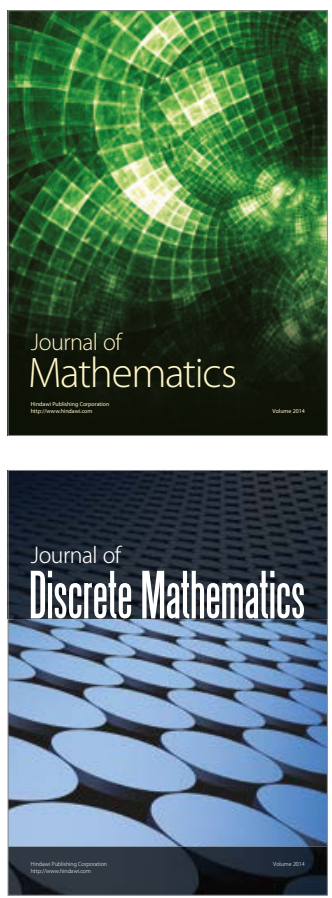

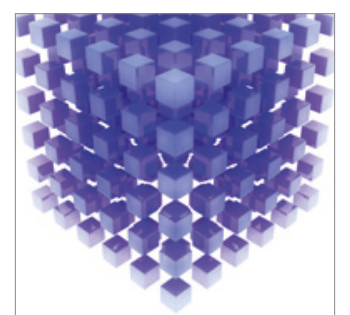

Mathematical Problems in Engineering
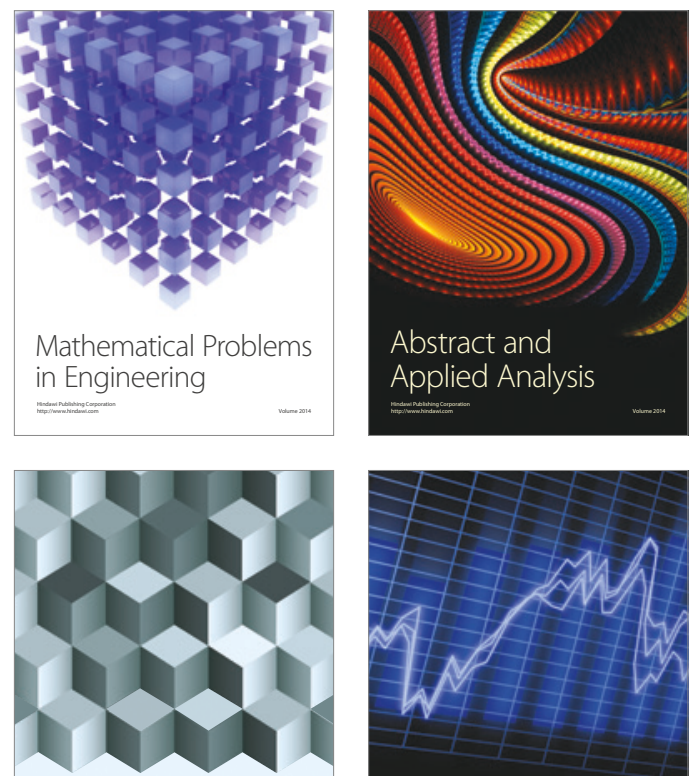

Journal of

Function Spaces

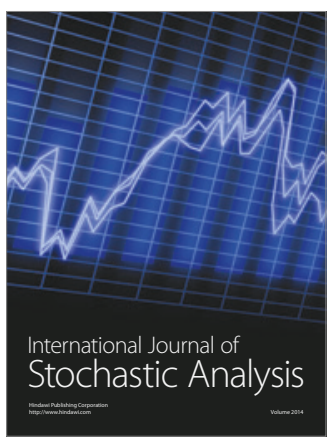

Probability and Statistics
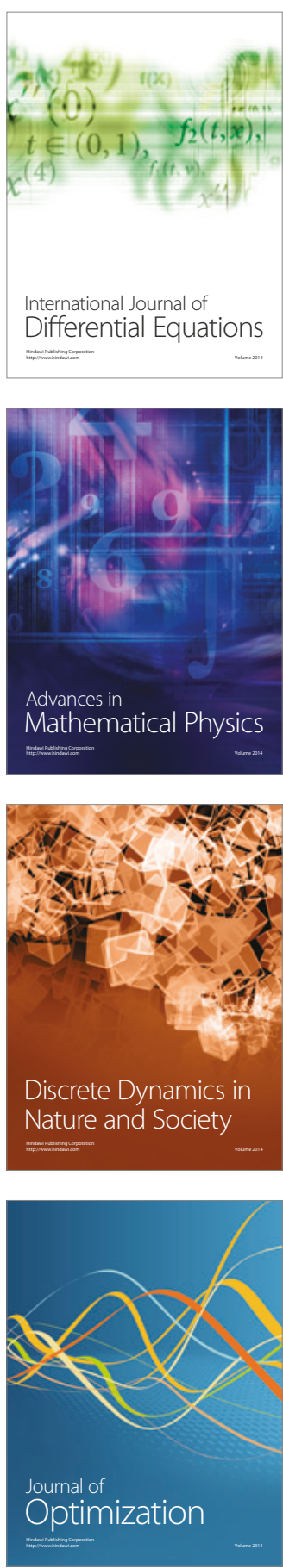